\title{
Agrobacterium rhizogenes-mediated hairy root transformation as an efficient system for gene function analysis in Litchi chinensis
}

\author{
Yaqi Qin, Dan Wang, Jiaxin Fu, Zhike Zhang, Yonghua Qin, Guibing Hu* and Jietang Zhao* (1)
}

\begin{abstract}
Background: Litchi chinensis Sonn. is an economically important fruit tree in tropical and subtropical regions. However, litchi functional genomics is severely hindered due to its recalcitrance to regeneration and stable transformation. Agrobacterium rhizogenes-mediated hairy root transgenic system provide an alternative to study functional genomics in woody plants. However, the hairy root transgenic system has not been established in litchi.

Results: In this study, we report a rapid and highly efficient $A$. rhizogenes-mediated co-transformation system in $L$. chinensis using Green Fluorescent Protein (GFP) gene as a marker. Both leaf discs and stem segments of $L$. chinensis Cv. 'Fenhongguiwei' seedlings were able to induce transgenic hairy roots. The optimal procedure involved the use of stem segments as explants, infection by A. rhizogenes strain MSU440 at optical density $\left(\mathrm{OD}_{600}\right)$ of 0.7 for 10 min and co-cultivation for 3 days, with a co-transformation efficiency of 9.33\%. Furthermore, the hairy root transgenic system was successfully used to validate the function of the key anthocyanin regulatory gene LCMYB1 in litchi. Over-expression of LCMYB1 produced red hairy roots, which accumulated higher contents of anthocyanins, proanthocyanins, and flavonols. Additionally, the genes involving in the flavonoid pathway were strongly activated in the red hairy roots.
\end{abstract}

Conclusion: We first established a rapid and efficient transformation system for the study of gene function in hairy roots of litchi using A. rhizogenes strain MSU440 by optimizing parameters. This hairy root transgenic system was effective for gene function analysis in litchi using the key anthocyanin regulator gene LCMYB1 as an example.

Keywords: Litchi chinensis, Agrobacterium rhizogenes, Hairy root, Anthocyanin, LCMYB1

\section{Background}

Litchi (Litchi chinensis Sonn.) is a perennial fruit tree of the Sapindaceae family cultivated in tropical and subtropical zones of the world. It has been cultivated for

\section{*Correspondence: guibing@scau.edu.cn; jtzhao@scau.edu.cn}

State Key Laboratory for Conservation and Utilization of Subtropical Agro-Bioresources/Key Laboratory of Biology and Genetic Improvement of Horticultural Crops (South China), Ministry of Agriculture and Rural Affairs/ Guangdong Litchi Engineering Research Center, College of Horticulture,

South China Agricultural University, Guangzhou, China more than 2300 years in China due to its delicious and nutritive fruits. In the past decade, substantial progress has been experienced into the molecular changes of flower and fruit development in litchi with the development of genetic and genomics tools [1-5]. However, gene functional characterization is severely hindered by the lack of an efficient regeneration and transformation protocol for litchi. Litchi is strong recalcitrance to in vitro regeneration. Different explants such as anthers, immature embryos, and leaves has been used to induce callus and somatic embryo, but only few of them regenerate successfully [6-8]. The main limitation of somatic embryogenesis is the large number of 
abnormal somatic embryos produced which cannot germinate or convert into normal plants [9].

Agrobacterium tumefaciens-mediated transformation has been widely used for plant genetic engineering and the study of gene function [10]. To date, successful A. tumefaciens-mediated transformation has been reported for several woody fruit crops, such as apple [11], peach [12], and citrus [13]. Puchooa [14] reported the $A$. tumefaciens-mediated litchi transformation protocols using leaf as explants, but no plantlets were regenerated from transformed calli. Padilla et al. [15] developed an A.tumefaciens-mediated transformation of 'Brewster' litchi with the PISTILLATA cDNA in antisense. In general, the transformation efficiency was low, and an efficient and reproducible transformation protocol is yet to be developed for litchi and other woody fruit crops [16].

A. rhizogenes-mediated hairy root transformation systems provide an alternative to species recalcitrant to transformation by A. tumefaciens [17]. Compared to $A$. tumefaciens-mediated transformation, A. rhizogenes-mediated transformation system is speedy, since transgenic hairy roots grow rapidly without a complex cultured process to regenerated plantlets $[18,19]$. Therefore, A. rhizogenes-mediated transformation constitutes a simple, rapid and efficient method for the production of metabolites and the study of gene function in plants [20]. In grapevine, hairy roots were used to study the function of $V v M y b P A 1$ or $V v M y b P A 2$ on the regulation of proanthocyanidin biosynthesis [21]. Recently, Meng et al. [17] developed a simple, fast and efficient $A$. rhizogenes-mediated transformation for generating stable transgenic roots in living plants to facilitate functional studies in vivo. Subsequently, one-step generation of composite plants with transgenic roots by $A$. rhizogenes-mediated transformation has been established in peach, cucumber, and soybean [22-25]. In sweet potato, A. rhizogenes-mediated in vivo root transgenic system were shown to be a suitable system for the functional characterization of genes involved in salt tolerance [25]. However, the hairy root system has not been established in litchi. Therefore, an efficient hairy root transformation protocol is needed for rapid characterization of gene function in litchi.

In this study, we developed a reproducible, rapid and highly efficient $A$. rhizogenes-mediated hairy root cotransformation system for litchi. We optimized the transformation procedure using Green Fluorescent Protein (GFP) gene as a marker. Finally, we demonstrated the potential of the hairy root transgenic system to enable gene functional studies in litchi using the key transcriptional factor $L c M Y B 1$ that regulates anthocyanin synthesis as an example.

\section{Materials and methods}

\section{Plant materials and culture media}

Seeds of Litchi chinensis Sonn. cv. 'Fenhongguiwei' were obtained from South China Agricultural University College, Guangzhou, China. Mature seeds were first washed in running water for $30 \mathrm{~min}$. Thoroughly washed seeds were surface-sterilized with $75 \%$ ethanol for $1 \mathrm{~min}$ and dipped in sodium hypochlorite solution (1\%) for $30 \mathrm{~min}$, then rinsed 5 times with sterile water. Surface sterilized seeds were cultured on 1/2 MS medium [26] without sucrose. Culture was maintained at $25{ }^{\circ} \mathrm{C}$ in full light conditions (3000 lx) on a $16 \mathrm{~h} / 8 \mathrm{~h}$ day/night cycle for seed germination.

\section{Agrobacterium strain and binary vectors}

Agrobacterium rhizogenes strain MSU440 was used to induce transgenic hairy roots in litchi. The coding sequence of LcMYB1 (accession number KY302802) without the termination codon was fused with the green fluorescent protein (eGFP) gene controlled by cauliflower mosaic virus (CaMV) 35S promoter in binary vector pCAMBIA1300 (Fig. 1). Binary vectors pCAMBIA1300eGFP and pCAMBIA1300-LcMYB1-eGFP were introduced into $A$. rhizogenes strain by freeze-thaw method [27].

\section{A. rhizogenes-mediated transformation}

A. rhizogenes strain MSU440 harboring binary vector pCAMBIA1300-eGFP or pCAMBIA1300-LcMYB1eGFP was cultured in $600 \mu \mathrm{L}$ YEP medium containing $50 \mathrm{mg} \mathrm{L}^{-1}$ kanamycin and $50 \mathrm{mg} \mathrm{L}^{-1}$ streptomycin and incubated overnight at $28{ }^{\circ} \mathrm{C}$ on a rotary shaker at $200 \mathrm{rpm}$. After the $\mathrm{OD}_{600}$ value reached to $0.6-0.8$, bacterial cells were centrifuged at $5000 \mathrm{rpm}$ for $8 \mathrm{~min}$ and re-suspended at different concentration $\left(\mathrm{OD}_{600}=0.3,0.5\right.$, $0.7,0.9)$ in MS liquid medium containing $100 \mu \mathrm{M}$ acetosyringone (AS). The resulting cell suspension culture was used in transformation.

Leaf discs and stem segments from 3- to 5- weeks-old litchi plants were submerged in bacterial solution for different incubation time $(10,20,30$, or $40 \mathrm{~min}$ ) (infection concentration $\mathrm{OD}_{600}=0.7$ ), followed by removal of

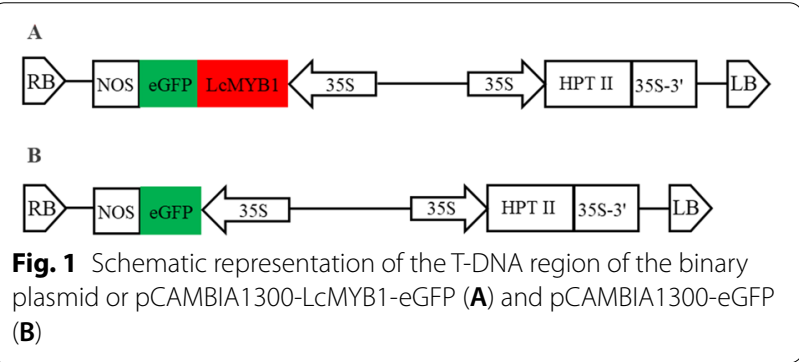


excessive liquid on sterile paper. Infected explants were then transferred onto filter papers wetted with the liquid MS medium with $100 \mu \mathrm{M}$ AS and co-cultivated in the dark for 3, 5, and 7 days. After co-cultivation, infected explants were washed with sterile water and transferred onto MS medium containing $300 \mathrm{mg} \mathrm{L}^{-1}$ timentin and $300 \mathrm{mg} \mathrm{L}^{-1}$ carbenicilin.

\section{Fluorescence assay of regenerated hairy roots}

The transgenic hairy roots were detected fluorescence using a stereomicroscope or scanning confocal microscopy (Zeiss, Germany) with filter sets for eGFP $(525 / 50 \mathrm{~nm})$. For histological assay of transgenic hairy roots, $0.5 \mathrm{~cm}$ long tip of hairy root was excised, and vertical sections were observed and imaged under a light microscope (DP27; Olympus, Japan).

\section{RNA isolation and PCR analysis}

Hairy root RNA was extracted using RNAprep pure Plant Kit (TIANGEN, China) following the instruction. Then $1 \mu \mathrm{g}$ total RNA was reverse-transcribed to cDNA using GoScript Reverse Transcription System (Promega, USA). RT-PCR was conducted using $2 \times$ Taq Master Mix (Vazyme, China) following manufacturer's instruction. Real-time quantitative PCR analysis
(RT-qPCR) with SYBR Green Master Mix (Vazyme, China) was run in ABI 7500 Real-Time PCR System (Applied Biosystems, USA). The primers were listed in the Table 1. LcActin (accession number HQ615689) was used as internal control. Relative expression levels of candidate genes were calculated with the formula $2^{-\Delta \Delta C t}$ [28]. All reactions were performed with three biological replicates.

\section{Analysis of anthocyanin, flavonols and proanthocyanidins content}

For anthocyanin quantitation, $50 \mathrm{mg}$ of hairy roots was extracted with a solution of mix methanol, water and concentrated hydrochloric acid (85:12:3) overnight at $4{ }^{\circ} \mathrm{C}$. Total anthocyanin content was measured using absorbance at $530 \mathrm{~nm}$ that have been diluted with $\mathrm{pH}$ 1.0 and 5.0 buffers.

For flavonols and proanthocyanidins extraction, hairy roots was extracted using methanol following the method described by Tiberti et al. [29] and Fiorella et al. [30], respectively. Flavonols and proanthocyanidins content were measured using absorbance at $510 \mathrm{~nm}$ and $500 \mathrm{~nm}$, respectively.

Table 1 List of primer sequence

\begin{tabular}{|c|c|c|}
\hline Gene & Forward primer $\left(5^{\prime}-3^{\prime}\right)$ & Reverse primer $\left(5^{\prime}-3^{\prime}\right)$ \\
\hline \multicolumn{3}{|c|}{ For real-time qPCR } \\
\hline LCMYB1 & ACAGCAGAGACCATTTAGGG & TGATGTTTGTCCAAGCAGTTC \\
\hline LCPAL & GCCAAGCAATTGATTTAAGG & CCACTTTGAGCAGATCCTTT \\
\hline $\mathrm{LCC} 4 \mathrm{H}$ & AGACGACTTGAACCACCGC & CCCGAACTCGACTCCCTGT \\
\hline LCCHS & GACATTGTGGTGGTGGAGGT & TATTTAGCGAGACGGAGGAC \\
\hline $\mathrm{LCCHI}$ & CGGAGTTTACTTGGAGGATGT & CAGTGACCTTCTCAGAGTATTG \\
\hline LCF3H & GGTGGATAGATGTGACAAAGGAGT & GGTTGTGGGCATTTTGGATAGTA \\
\hline LCF3'H & GCTCCGTCCATCTCTTCTCC & CCGTCTCCGAACACTCTCC \\
\hline LCDFR & GGACCCTGAAAACGAAGTAA & CACTCCAGCAAGTCTCATCA \\
\hline LCANS & AGGAAGTTGGTGGTCTGGAAG & CCGTTGCTGAGGATTTCAATGGTG \\
\hline LCUFGT & GCCACCAGCGGTTCCTAATA & ATGCCTCTGCTACTGCTACAATCT \\
\hline LCGST & GAGCATAAGCGTCCTGAGTTTC & TCCACGGTCCGCATACTTG \\
\hline LCLAR1 & TGAGAGTAGAGAAATCCGAATG & ATGACCTGTTTGGTAGAGAGAA \\
\hline LCLAR2 & ATGGCACCGTCAAAGCATAC & TTTCCCACAGAGAAGCAAGC \\
\hline LCANR & AGGGCTATGTTGTTCACACTAC & AGCAAAATTGACTGGTGTTG \\
\hline LCFLSI & GAGAGAGGTGGTGGACAAGT & TTGGAACAAGAACGGTGAG \\
\hline LCFLS2 & AGCCCATTGAAGGTGTAAAG & CTTGGAGCCGTTGGATTA \\
\hline LCACT & ACCGTATGAGCAAGGAAATCACTG & TCGTCGTACTCACCCTTTGAAATC \\
\hline \multicolumn{3}{|c|}{ For analysis of PCR } \\
\hline eGFP & ATGGTGAGCAAGGGCGAGGAGCTGTTCACC & TTACTTGTACAGCTCGTCCATGCCGAGAGTGATCCC \\
\hline $\mathrm{rol} B$ & GCTCTTGCAGTGCTAGATTT & GAAGGTGCAAGCTACCTCTC \\
\hline hpt II & СTATTTCTTTGCCCTCGGACGAGTGCTGGGGCGT & ATGAAAAAGCCTGAACTCACCGCGACGTCTGTCGA \\
\hline $\operatorname{Vir} D$ & ATGTCGCAAGGCAGTAAG & CAAGGAGTCTTTCAGCATG \\
\hline
\end{tabular}




\section{Statistical analysis}

All experiment were conducted with three times. All the statistical analysis were performed using a $t$-test by SPSS software. Significance was indicated by asterisks * $(P<0.05)$.

\section{Results}

\section{Transgenic hairy root induction from litchi}

Leaves and stems from 4-week-old seedlings were used as transformation explants. Approximately 20 days after infection, calli appeared around the cutting site of leaves and stems (Fig. 2A, C). After about 5 weeks, small hairy roots appeared from the calli and elongated (Fig. 2E, G). The co-transgenic roots were detected by screening for GFP fluorescence signal. GFP expression was initially weak and only observed in a few cells of the calli from leaf and stem explants (Fig. 2B, D). Later, stronger GFP expression was observed in the entire transgenic hairy roots (Fig. 2F, H). To further verify that the co-transgenic nature of hairy roots regenerated, PCR analysis was carried out from 4 randomly selected independent transgenic hairy roots. Bands representing the $e G F P$ and $h p t I I$ were detected in the transgenic hairy roots with GFP fluorescence, but not in the control (Fig. 2I). Similarly, the $\operatorname{rol} B$ gene responsible for directing hair roots differentiation was also detected in transgenic hairy roots. In addition, Vir $D$ gene required for the T-DNA transfer and processing but located outside of T-DNA region of Ri plasmid was absent in transgenic hairy roots (Fig. 2I), showing that there was no A. rhizogenes contamination. In summary, a successful $A$. rhizogenes-mediated hairy roots cotransformation system was established in litchi.
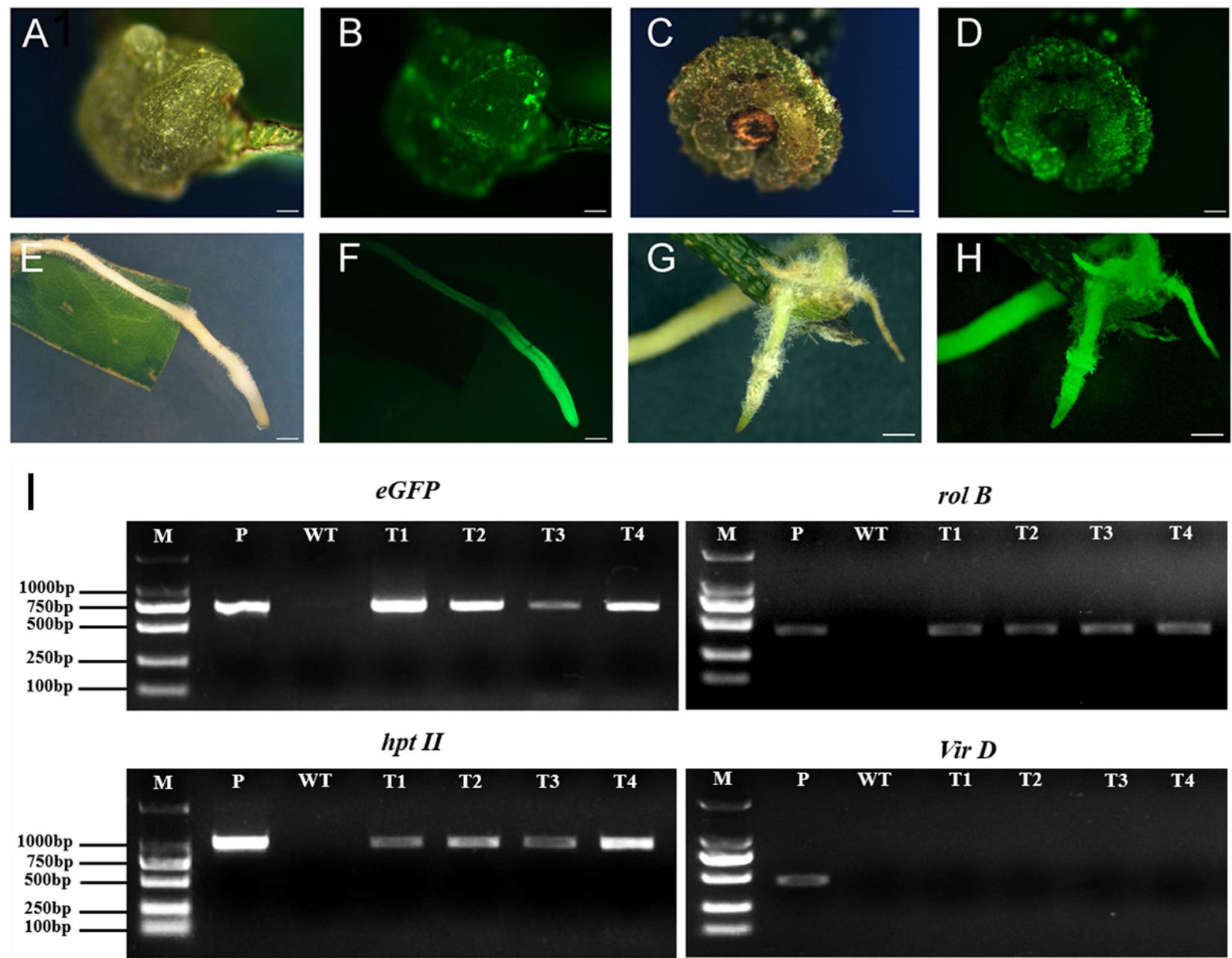

Fig. 2 A. rhizogenes mediated transformation of litchi. Calli (A) and GFP fluorescence (B) induced from leaf discs. Calli (C) and GFP fluorescence (D) induced from stem fragments. Transgenic hairy root $(\mathbf{E})$ and GFP fluorescence $(\mathbf{F})$ from leaf explants. Transgenic hairy root $(\mathbf{G})$ and GFP fluorescence (H) from stem explants. PCR analysis (I) for eGFP, rol B, hpt II, and Vir D in independent transgenic hairy roots 


\section{Optimization of A. rhizogenes-mediated litchi transformation}

In order to optimize the $A$. rhizogenes co-transformation efficiency, A. rhizogenes concentration, infection time, and duration of co-cultivation were tested. Firstly, various concentrations of $A$. rhizogenes were compared with the infection time of $10 \mathrm{~min}$ and co-cultivation for 3 days. When A. rhizogenes concentration of $\mathrm{OD}_{600}$ value ranged from 0.3 to 0.9 , the hairy root regeneration rate of leaf and stem explants ranged from 35 to $48 \%$, but there was no significant difference between leaf and stem explants (Fig. 3A). The highest co-transformation efficiency of leaf and stem were $8.33 \%$ and $8.89 \%$, respectively (Fig. 3D). Subsequently, different infection times were tested with the $A$. rhizogenes concentration of $\mathrm{OD}_{600}=0.7$ and cocultivation for 3 days. The hairy root regeneration rate of leaf and stem explants decreased significantly with the increasing infection times (Fig. 3B). Co-transformation efficiency increased significantly from 5 to $10 \mathrm{~min}$, then decreased significantly. The co-transformation efficiency in $10 \mathrm{~min}$ of leaf and stem were $6.67 \%$ and $8.89 \%$, respectively (Fig. 3E). Finally, various co-cultivation times were evaluated with the $A$. rhizogenes concentration of $\mathrm{OD}_{600}=0.7$ and infection time for $10 \mathrm{~min}$. The results indicated that the hairy root regeneration rate and cotransformation efficiency of leaf and stem at the 2 days and 3 days co-cultivation duration was significantly higher than that of 4 days and 5 days (Fig. 3C, F). When co-cultivation duration for 3 days, the highest co-transformation efficiency of leaf and stem were $5.56 \%$ and $9.33 \%$, respectively. Taken together, these results indicated the optimal infection condition was $A$. rhizogenes concentration of $\mathrm{OD}_{600}=0.7$, infection time for $10 \mathrm{~min}$, and co-cultivation for 3 days.

\section{Gene functional analysis using the hairy root transgenic system}

To further confirm that the hairy root transgenic system is suitable for conducting gene functional analysis, $L c M Y B 1$, the key transcription factor that regulates anthocyanin biosynthesis in litchi, was co-transformed in the hairy root system. The coding sequence was cloned into pCAMBIA1300-eGFP vector to generate the C-terminal GFP fusion protein (Fig. 1A). Then the pCAMBIA1300-LcMYB1-eGFP vector was transferred into A. rhizogenes strain MSU440 and used for transformation. Red callus appeared after 3 weeks of overexpressing $L c M Y B 1$ in leaf and stem using the hairy root system (Fig. 4A). After 1 more week, red hairy roots were regenerated from the red callus (Fig. 4B, C). PCR analysis revealed that LcMYB1, eGFP, hpt II, and Vir D genes
A

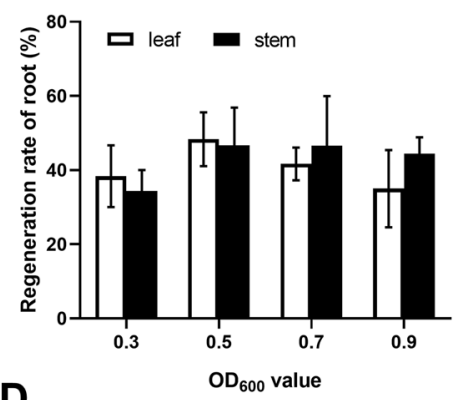

D

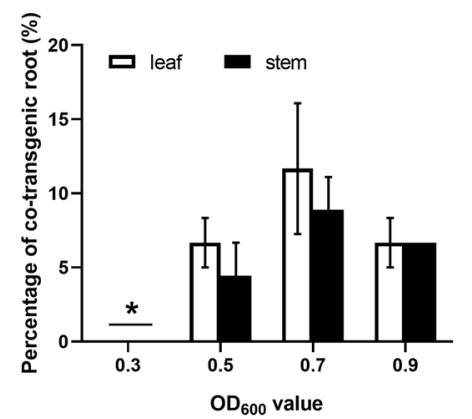

B
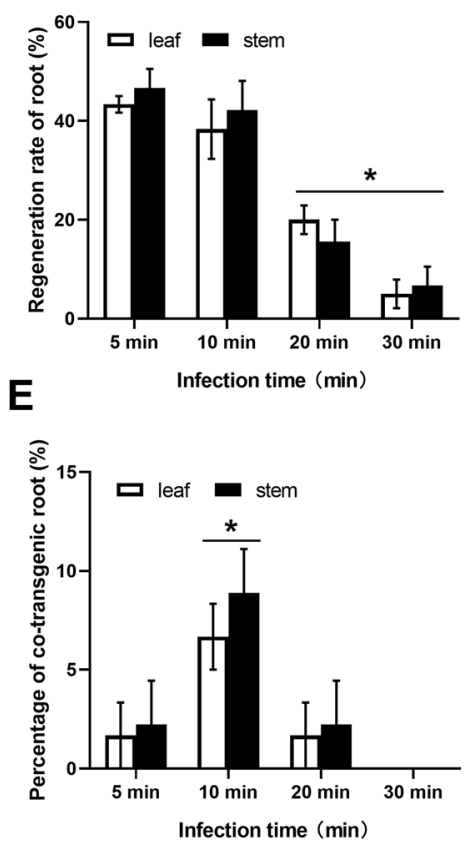

C
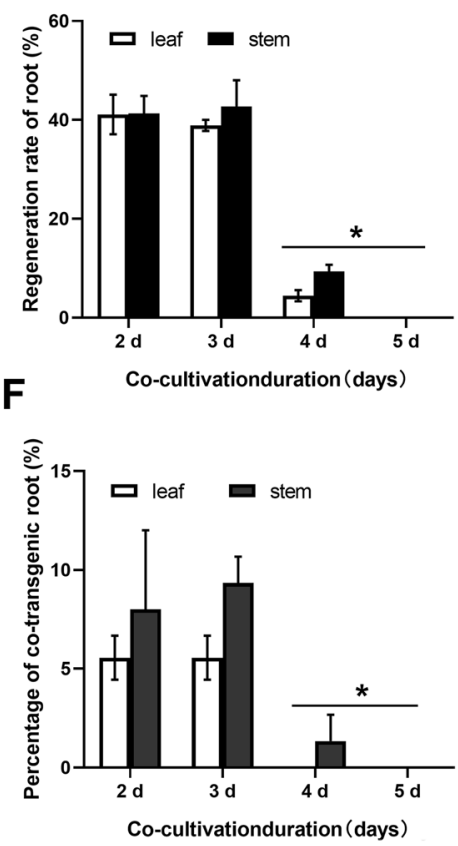

Fig. 3 Optimization of $A$. rhizogenes-mediated litchi transformation using leaf and stem as explants. The regeneration rate of hairy roots (A-C) and co-transformation efficiency (D-F) after infecting different $\mathrm{OD}_{600}$ values, times, and co-cultivation duration of Agrobacterium strain MSU440, respectively. * indicate significant differences $(P<0.05)$ 

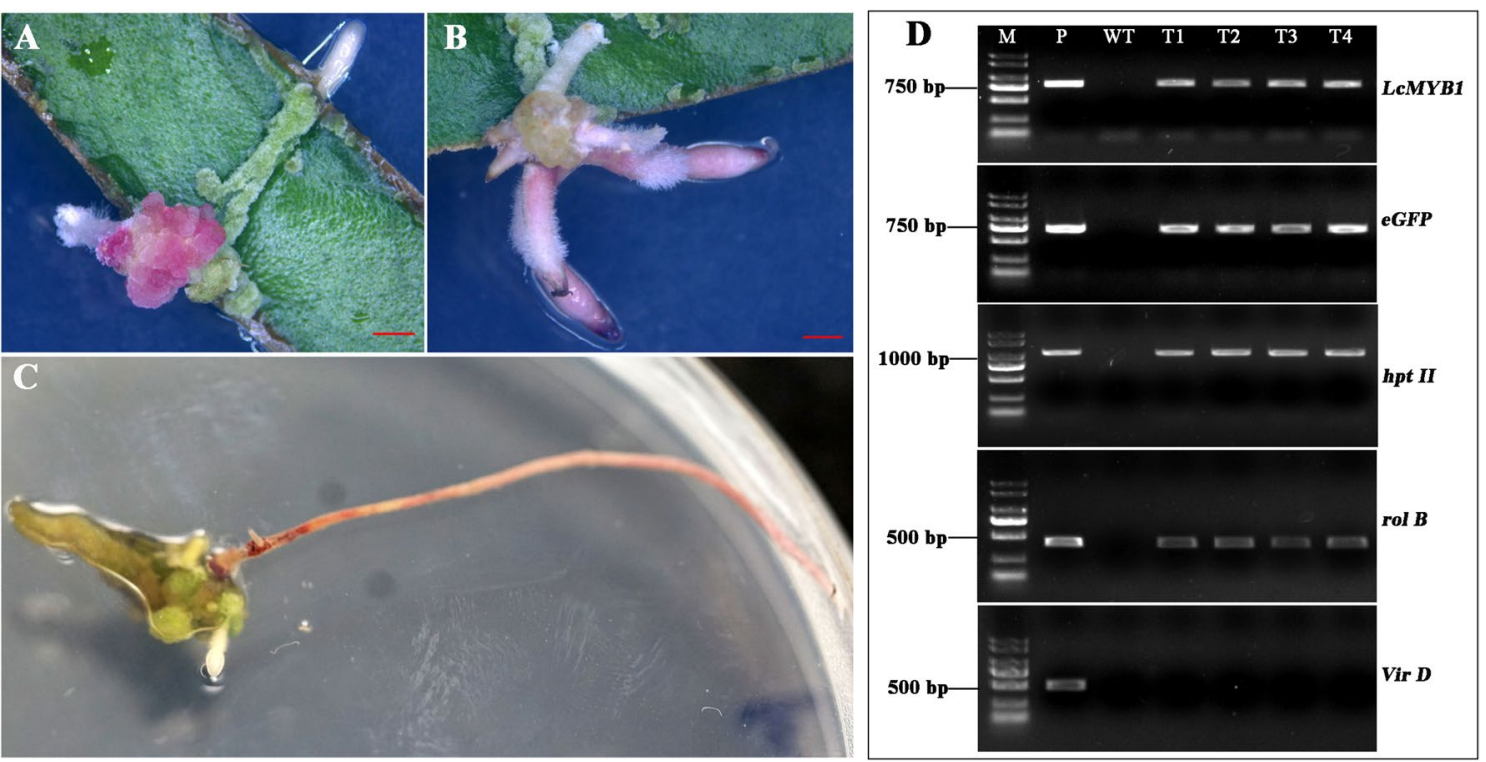

Fig. 4 Phenotype and molecular validation of transgenic hairy roots overexpressing LCMYB1 in leaf discs. Callus (A) and transgenic hairy roots (B, C) induced from leaf explants. PCR analysis (D) of LCMYB1, eGFP, rol B, hpt Il, and Vir D in independent transgenic hairy roots for validation of overexpressing LCMYBI
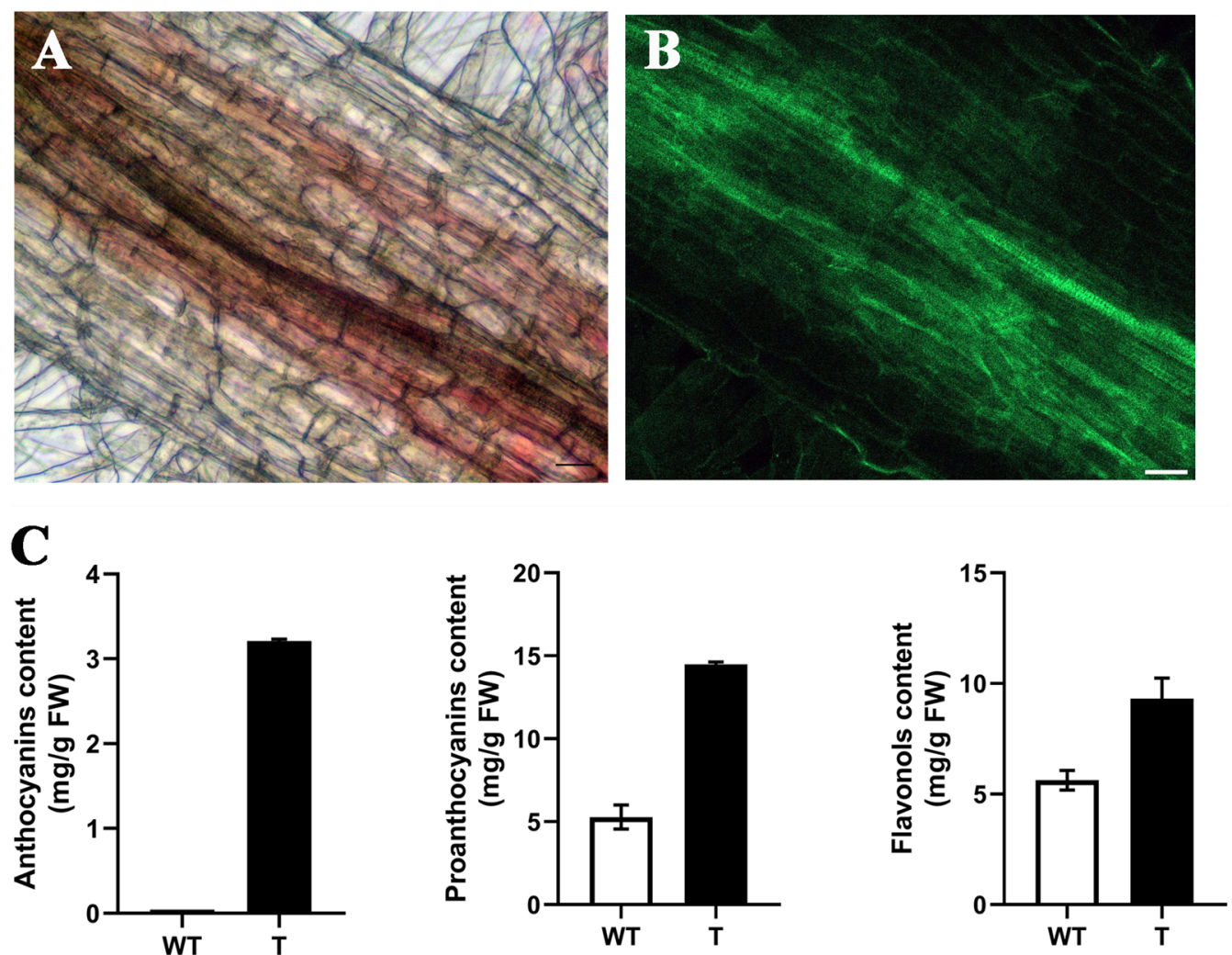

Fig. 5 Color observation and quantitative analysis of anthocyanins, proanthocyanins, and flavonols contents in red hairy roots. Red coloration (A) was seen in the transgenic hairy roots exhibiting GFP expression (B). C Relative anthocyanin, proanthocyanins, and flavonols contents of wild-type (WT) and transgenic red hairy roots ( $\mathrm{T}$ ) 
were detected in the red hairy roots, but absent in the control (Fig. 4D).

Upon closer inspection, the cells of red hairy roots were visualized in red by accumulating anthocyanin (Fig. 5A) and all the red cells exhibited stable GFP expression (Fig. 5B). In contrast, anthocyanins and GFP expression were absent in the non-transgenic roots. Quantitative analysis showed that contents of anthocyanins, proanthocyanins, and flavonols were significantly higher in red hairy roots. The relative anthocyanins, proanthocyanins, and flavonols contents in red hairy roots were, respectively, 69, 3, and 2 times greater than the control roots (Fig. 5C).

To confirm the role of LcMYB1, the expression levels of its target genes in the flavonoid pathway, including LcPAL (LITCHI018600), LcC4H (LITCHI031057), Lc4CL (LITCHI002917), LcCHS (LITCHI020852), LcCHI (LITCHI027959), LcF3H (LITCHI006477), LcF3'H (LITCHI023381), LcDFR (LITCHI010261), LcANS (LITCHI022925), LcUFGT (LITCHI002457), LcGST (LITCHI008070), LcFLS1 (LITCHI007338), LcFLS2 (LITCHI011187), LcLAR1 (LITCHI028570), LcLAR2 (LITCHI005474), and LcANR (LITCHI029356) were detected using RT-qPCR. The results indicated that the expression of all these genes were up-regulated (Fig. 6). LcGST4 (accession number KT946768), the gene involved in anthocyanin sequestration in litchi, was up-regulated more than 75281 folds. Additionally, the expression levels of $L c F L S 1 / 2$ involving in flavonols synthesis and $L c L A R 1 / 2$ and $L c A N R$ involving in proanthocyanidins synthesis were also up-regulated. Taken together, these results confirmed that the transgenic hairy roots system mediated by $A$. rhizogenes could be used to study anthocyanin metabolism in litchi and offer a simple way to verify gene function in woody plants.

\section{Discussion}

In the last two decades, increasing fruit tree genomic resources like genome sequences were available paving the way to the genetic engineering and molecular breeding of fruit plants for crop improvement [31]. It will be possible to identify the genes controlling the important horticultural traits. In litchi, transcriptome analysis was used to identify the key genes involving in anthocyanin biosynthesis [2, 32]. However, their function analysis was usually validated in model plants such as Arabidopsis and tobacco $[4,33,34]$. The major reason for this is the lack of litchi transformation system [35]. An effective transgenic technology will be crucial for genes functional analysis. Thus, our newly developed transgenic hairy roots system mediated by $A$. rhizogenes will advance functional genomics research in litchi.

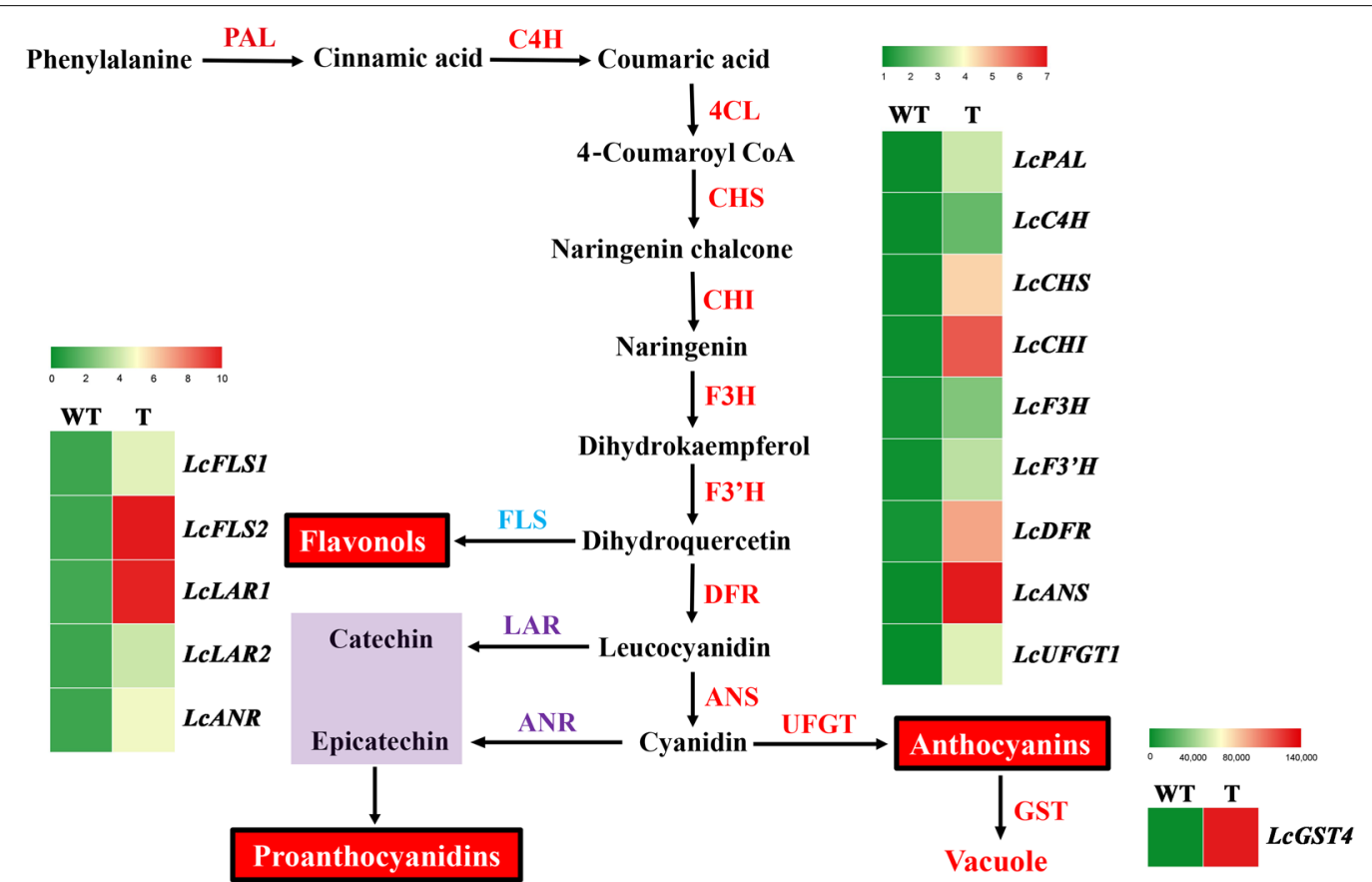

Fig. 6 The expression levels of key enzyme genes involved in flavonoid pathway analysed using RT-qPCR were shown in hotspot map. PAL phenylalanine ammonia lyase, C4H cinnamate-4-hydroxylase, 4CL 4-coumaroyl-coA synthase, CHS chalcone synthase, CHI chalcone-flavanone isomerase, F3H flavanone-3-hydroxylase, F3' H flavonoid-3'-hydroxylase, FLS flavonol synthase, DFR dihydroflavonol-4-reductase, LAR leucoanthocyanidin reductase, ANR anthocyanidin reductase, UFGT UDP-glucose:flavonoid-3-Oglucosyltransferase, GST glutathione-S-transferase 
To date, A. tumefaciens-mediated transformation systems were widely used for functional genomics in plants [10]. Recently, significant advances have been made in optimizing the genetic transformation of fruit trees [36, 37]. In litchi, only one report was successful in developing transgenic plants using $A$. tumefaciens-mediated method [15]. In this report, only gene expression levels of the four transgenic lines were analyzed without any phenotype analysis, as it needs $7-8$ years (typical juvenile period for litchi) to obtain mature plants [15]. Therefore, A. tumefaciens-mediated transformation is time-consuming, recalcitrant nature and not efficient enough to allow the high-throughput for functional genomic research [23]. Besides A. tumefaciens, A. rhizogenes is also utilized on functional studies of genes, especially those involved in secondary metabolism and responses to environmental stresses [20]. Here, A. rhizogenes-mediated transformation was established in litchi using leaf and stem as explants. Similar report also showed that hypocotyl, leaf and shoot were suitable for $A$. rhizogenes-mediated transformation in peach [22].

Factors including Agrobacterium concentration, infection time, and co-cultivation time affect T-DNA delivery and its integration into the plant genome $[38,39]$. In the present study, the co-transformation efficiency for leaf and stem explants were higher after they were co-cultivated for 2 or 3 days. With a longer period of 4 or $5 \mathrm{~d}, A$. rhizogenes may overgrow, leading to explant cell damage, consequently, to low transformation efficiency [18]. There was no significantly difference of co-transformation efficiency for leaf and stem explants in litchi. Liu et al. [18] reported that the $A$. rhizogenes transformation efficiency varied with the type of explant of Arachis hypogaea and was highest with petioles.

So far, transgenic hairy roots system mediated by $A$. rhizogenes has already been widely applied for many purposes including metabolism, root biology, and stress response [20, 25, 40]. Here, we used the established transgenic hairy roots system to study the gene function of $L c M Y B 1$ which regulates anthocyanin biosynthesis in litchi. The result indicated that overexpression of $L c M Y B 1$ could induce anthocyanin accumulation and produce red hairy roots. Previously, anthocyanins were accumulated in the tobacco hairy roots overexpressing $L c M Y B 1$ [41]. RT-qPCR showed that $L c M Y B 1$ could induce the expression of structural genes involved in anthocyanin biosynthetic in hairy roots of litchi. Therefore, transgenic hairy roots system was validated as an effective overexpression technique to study gene function in litchi. In addition, transgenic root system allows for silencing techniques or gene editing to be applied to plants that are recalcitrance to in vitro regeneration
$[17,42]$. These techniques in litchi need to be further explored.

\section{Conclusion}

Here, we first established a rapid and efficient root transgenic system for litchi. The optimal parameters were infection by $A$. rhizogenes strain MSU440 at $\mathrm{OD}_{600}$ of 0.7 for $10 \mathrm{~min}$ and co-cultivation for 3 days. We validated this transformation system for study gene function in transgenic hairy roots by producing transgenic roots overexpressing $L c M Y B 1$, the key transcription factor that regulates anthocyanin biosynthesis in litchi. Transgenic roots demonstrated red color and increased flavonoid contents and displayed upregulation of flavonoid-related genes. These results will help to provide a simple and rapid method for gene function analysis in litchi.

\section{Acknowledgements}

Not applicable.

\section{Authors' contributions}

JZ carried out conception of the research, and wrote the manuscript. YQ, DW and JZ performed the experiments. JF, ZZ, and YQ gave the critical suggestion of this study and revised the manuscript. GH supervised the entire study. All authors read and approved the final manuscript.

\section{Funding}

This work was funded by the National Key Research and Development Program (No. 2019YFD1000900), the National Natural Science Fund of China (No. 31872066), China Litchi and Longan Industry Technology Research System (No. CARS-32-05), the Science and Technology Planning Project of Guangzhou (No. 202103000057), and the Science and Technology Talents and Platform Plan of Yunnan Province (No. 202104AC100001-B04).

Availability of data and materials

Not applicable.

\section{Declarations}

Ethics approval and consent to participate

Not applicable.

Consent for publication

Not applicable.

Competing interests

The authors have no competing interests to declare.

Received: 19 July 2021 Accepted: 26 September 2021

Published: 9 October 2021

References

1. Zhang HN, Wei YZ, Shen JY, Lai B, Huang XM, Ding F, et al. Transcriptomic analysis of floral initiation in litchi (Litchi chinensis Sonn.) based on de novo RNA sequencing. Plant Cell Rep. 2014;33:1723-35.

2. Lai B, Hu B, Qin Y, Zhao J, Wang H, Hu G. Transcriptomic analysis of Litchi chinensis pericarp during maturation with a focus on chlorophyll degradation and flavonoid biosynthesis. BMC Genomics. 2015;16:225.

3. Zhang J, Wu Z, Hu F, Liu L, Huang X, Zhao J, et al. Aberrant seed development in Litchi chinensis is associated with the impaired expression of cell wall invertase genes. Hortic Res. 2018;5:39. 
4. Hu B, Lai B, Wang D, Li J, Chen L, Qin Y, et al. Three LCABFs are involved in the regulation of chlorophyll degradation and anthocyanin biosynthesis during fruit ripening in Litchi chinensis. Plant Cell Physiol. 2019;60:448-61.

5. Zhao M, Li J. Molecular events involved in fruitlet abscission in litchi. Plants. 2020;9:151.

6. Raharjo SHT, Litz RE. Somatic embryogenesis and plant regeneration of litchi (Litchi chinensis Sonn.) from leaves of mature phase trees. Plant Cell Tiss Organ Cult. 2007;89:113-9.

7. Ma XY, Yi GJ, Huang XL, Zeng J. Leaf callus induction and suspension culture establishment in lychee (Litchi chinensis Sonn.) cv Huaizhi. Acta Physiol Plant. 2009;31:401-5.

8. Wang G, Li H, Wang S, Sun J, Zhang X, Wang J. In vitro regeneration of Feizixiao litchi (Litchi chinensis Sonn.). Afr J Biotechnol. 2016;15:1026-34.

9. Garcia C, Furtado de Almeida AA, Costa M, Britoo D, Valle R, Royaert S, et al. Abnormalities in somatic embryogenesis caused by 2,4-D: an overview. Plant Cell Tiss Organ Cult. 2019;137:193-212.

10. Vain P. Thirty years of plant transformation technology development. Plant Biotechnol J. 2007:5:221-9.

11. Yao JL, Cohen D, Atkinson R, Richardson K, Morris B. Regeneration of transgenic plants from the commercial apple cultivar Royal Gala. Plant Cell Rep. 1995; 14:407-12.

12. Perez-Clemente RM, Perez-Sanjuan A, Garcia-Ferriz L, Beltran JP, Canas LA Transgenic peach plants (Prunus persica L.) produced by genetic transformation of embryo sections using the green fluorescent protein (GFP) as an in vivo marker. Mol Breed. 2004;14:419-27.

13. Dutt M, Madhavaraj J, Grosser JW. Agrobacterium tumefaciens-mediated genetic transformation and plant regeneration from a complex tetraploid hybrid citrus rootstock. Sci Hortic. 2010;123:454-8.

14. Puchooa D. Expression of green fluorescent protein gene in litchi (Litchi chinensis Sonn.) tissues. J Appl Hort. 2004;6:11-5.

15. Padilla G, Pérez JA, Perea-Arango I, Moon PA, Gómez-Lim MA, Borges AA et al. Agrobacterium tumefaciens-mediated transformation of "Brewster" ('Chen Tze') litchi (Litchi chinensis Sonn.) with the PISTILLATA cDNA in antisense. In Vitro Cell Dev Biol Plant. 2013;49:510-9.

16. Song G, Prieto H, Orbovic V. Agrobacterium-mediated transformation of tree fruit crops: methods, progress, and challenges. Front Plant Sci. 2019;10:226.

17. Meng D, Yang Q, Dong B, Song Z, Niu L, Wang L, et al. Development of an efficient root transgenic system for pigeon pea and its application to other important economically plants. Plant Biotechnol J. 2019;17:1804-13.

18. Liu S, Su L, Liu S, Zeng X, Zheng D, Hong L, et al. Agrobacterium rhizogenes-mediated transformation of Arachis hypogaea: an efficient tool for functional study of genes. Biotechnol Biotec Eq. 2016;30:869-78.

19. Gomes C, Dupas A, Pagano A, Grima-Pettenati J, Paiva JAP. Hairy root transformation: a useful tool to explore gene function and expression in Salix spp. recalcitrant to transformation. Front Plant Sci. 2019;10:1427.

20. Veena V, Taylor CG. Agrobacterium rhizogenes: recent developments and promising applications. In Vitro Cell Dev Biol -Plant. 2007;43:383-403.

21. Terrier N, Torregrosa L, Ageorges A, Vialet S, Verriès C, Cheynier V, et al. Ectopic expression of VVMybPA2 promotes proanthocyanidin biosynthesis in grapevine and suggests additional targets in the pathway. Plant Physiol. 2009;149:1028-41.

22. Xu S, Lai E, Zhao L, Cai Y, Ogutu C, Cherono S, et al. Development of a fast and efficient root transgenic system for functional genomics and genetic engineering in peach. Sci Rep. 2020;10:2836.

23. Fan $Y$, Xu F, Zhou H, Liu X, Yang X, Weng K, et al. A fast, simple, high efficient and one-step generation of composite cucumber plants with transgenic roots by Agrobacterium rhizogenes-mediated transformation. Plant Cell Tiss Organ Cult. 2020;141:207-16.

24. Fan $Y$, Zhang $X$, Zhong L, Wang $X$, Jin L, Lyu S. One-step generation of composite soybean plants with transgenic roots by Agrobacterium rhizogenes-mediated transformation. BMC Plant Biol. 2020;20:208.

25. Yu Y, Xuan Y, Bian X, Zhang L, Pan Z, Kou M, et al. Overexpression of phosphatidylserine synthase IbPSS1 affords cellular $\mathrm{Na}^{+}$homeostasis and salt tolerance by activating plasma membrane $\mathrm{Na}^{+} / \mathrm{H}^{+}$antiport activity in sweet potato roots. Hortic Res. 2020;7:131.

26. Murashige T, Skoog F. A revised medium for rapid growth and bioassays with tobacco tissue cultures. Physiol Plant. 1962;15:473-97.
27. Holsters M, de Waele D, Depicker A, Messens E, van Montagu M, Schell J. Transfection and transformation of Agrobacterium tumefaciens. Molec Gen Genet. 1978;163:181-7.

28. Livak KJ, Schmittgen TD. Analysis of relative gene expression data using real-time quantitative $P C R$ and the $2-\triangle \triangle C T$ method. Methods. 2001;25:402-8.

29. Tiberti LA, Yariwake JH, Ndjoko K, Hostettmann K. On-line LC/UV/MS analysis of flavonols in the three apple varieties most widely cultivated in Brazil. J Brazil Chem Soc. 2007:18:100-5.

30. Cerpa-Calderon FK, Kennedy JA. Berry integrity and extraction of skin and seed proanthocyanidins during red wine fermentation. J Agric Food Chem. 2008:56:9006-14.

31. Rai MK, Shekhawat NS. Genomic resources in fruit plants: an assessment of current status. Crit Rev Biotechnol. 2015:35:438-47.

32. Hu B, Li J, Wang D, Wang H, Qin Y, Hu G, et al. Transcriptome profiling of Litchi chinensis pericarp in response to exogenous cytokinins and abscisic acid. Plant Growth Regul. 2018;84:437-50.

33. Hu B, Zhao J, Lai B, Qin Y, Wang H, Hu G. LCGST4 is an anthocyanin-related glutathione S-transferase gene in Litchi chinensis Sonn. Plant Cell Rep. 2016;35:831-43.

34. Ma A, Wang D, Lu H, Wang H, Qin Y, Hu G, et al. LcCOP1 and LcHY5 contro the suppression and induction of anthocyanin accumulation in bagging and debagging litchi fruit pericarp. Sci Hortic. 2021;287:110281.

35. Dalei K, Sahu BB, Kumari M, Tripathi RM, Pudake RN. Advances in genetic transformation of Litchi. In: Kumar M, Kumar V, Prasad R, Varma A, editors. The Lychee biotechnology. Singapore: Springer; 2017. p. 421-36.

36. Gambino G, Gribaudo I. Genetic transformation of fruit trees: current status and remaining challenges. Transgenic Res. 2012;21:1163-81.

37. Rai MK, Shekhawat NS. Recent advances in genetic engineering for improvement of fruit crops. Plant Cell Tiss Organ Cult. 2014;116:1-15.

38. Ziemienowicz A. Agrobacterium-mediated plant transformation: factors, applications and recent advances. Biocatal Agric Biotechnol. 2014:3:95-102.

39. Zhao J, Li Z, Henny RJ, Gray DJ, Xie J, Chen J. Efficient somatic embryogenesis and Agrobacterium-mediated transformation of Epipremnum aureum'Jade.' Plant Cell Tiss Org. 2013;114:237-47.

40. Kereszt A, Li D, Indrasumunar A, Nguyen C, Nontachaiyapoom S, Kinkema $\mathrm{M}$, et al. Agrobacterium rhizogenes-mediated transformation of soybean to study root biology. Nat Protoc. 2007;2:948-52.

41. Qin Y, Hu G, Zhao J. Studies on Agrobacterium rhizogenesis-mediated transformation of LCMYB1 gene into tobacco leaves. Yuan Yi Xue Bao. 2020:47:635-42.

42. Cai Y, Chen L, Liu X, Sun S, Wu C, Jiang B, et al. CRISPR/Cas9-mediated genome editing in soybean hairy roots. PLoS ONE. 2015:10:e0136064.

\section{Publisher's Note}

Springer Nature remains neutral with regard to jurisdictional claims in published maps and institutional affiliations.

Ready to submit your research? Choose BMC and benefit from

- fast, convenient online submission

- thorough peer review by experienced researchers in your field

- rapid publication on acceptance

- support for research data, including large and complex data types

- gold Open Access which fosters wider collaboration and increased citations

- maximum visibility for your research: over 100M website views per year

At BMC, research is always in progress.

Learn more biomedcentral.com/submissions 